\title{
Methods to isolate extracellular vesicles for diagnosis
}

\author{
Hyejin Kang ${ }^{1}$, Jiyoon Kim ${ }^{1}$ and Jaesung Park ${ }^{1,2^{*}}$
}

\begin{abstract}
Extracellular vesicles (EVs) are small membrane-bound bodies that are released into extracellular space by diverse cells, and are found in body fluids like blood, urine and saliva. EVs contain RNA, DNA and proteins, which can be biomarkers for diagnosis. EVs can be obtained by minimally-invasive biopsy, so they are useful in disease diagnosis. High yield and purity contribute to precise diagnosis of disease, but damaged EVs and impurities can cause confused results. However, EV isolation methods have different yields and purities. Furthermore, the isolation method that is most suitable to maximize EV recovery efficiency depends on the experimental conditions. This review focuses on merits and demerits of several types of EV isolation methods, and provides examples of how to diagnose disease by exploiting information obtained by analysis of EVs.
\end{abstract}

Keywords: Extracellular vesicle (EV), Ultracentrifugation (UC), Immunoaffinity, Size exclusion, Polymer precipitation, Microfluidics techniques, Diagnosis

\section{Background}

Extracellular vesicles (EVs) are nanometer-to-micrometer sized vesicles that are released by cells [1]. EVs occur in several forms that differ in dimension, mechanism of production, and cellular origin (Fig. 1) [2]. According to mechanism of production, EVs are classified as exosomes, microvesicles, and apoptotic bodies [3, 4]. Exosomes arise from early endosomes [5]: as intraluminal vesicles formed by invagination of endosomal membranes, early endosomes come to have several intraluminal vesicles and become multivesicular bodies (MVBs) $[4,6]$. When MVBs fuse with plasma membranes, vesicles within MVBs are released into the extracellular environment and are called exosomes [4]. Exosomes are 40-100 nm in diameter and generally bear surface markers such as tetraspanins (CD9, CD81, CD63), Alix, or TSG101 [7]. Microvesicles, sometimes called ectosomes, are generated by outward budding or evagination of plasma membranes into extracellular space; they carry cytoplasmic

\footnotetext{
*Correspondence: jpark@postech.ac.kr

${ }^{1}$ School of Interdisciplinary Bioscience and Bioengineering, POSTECH, 77

Cheongam-Ro, Pohang, Gyeongbuk 37673, Republic of Korea

Full list of author information is available at the end of the article
}

contents. Microvesicles do not arise from the endosomal pathway.

Characteristics of exosomes and microvesicles do not clearly distinguish them. Some studies have tried to discriminate exosome and microvesicle based on origin, size and density [8]. Microvesicles are larger (100-1000 nm) than exosomes $(40-100 \mathrm{~nm})$ [9]. The size and number of EVs are normally determined by nanoparticle tracking analysis (NTA) or tunable resistive pulse sensing (TRPS) [10]. Exosomes and microvesicles can be distinguished based on size, but cannot be easily distinguished based on only protein markers on the vesicle membrane. Tetraspanins, which are commonly used to define EVs, are enriched in both exosomes and microvesicles [11, 12]. Distinction between the two classes requires more-accurate clues than tetraspanin presence.

Apoptotic bodies are produced by dying cells as they disintegrate [13, 14]; the bodies are diverse in size (generally 50-5000 nm) [15]. However, this classification based on biogenesis can cause confusion, because small microvesicles that arise from evagination of plasma membranes are also called exosomes in some cases, and because different groups of EVs cannot be completely discriminated during isolation $[5,16]$. Sometimes EVs are named after the cells that released them; for example, 


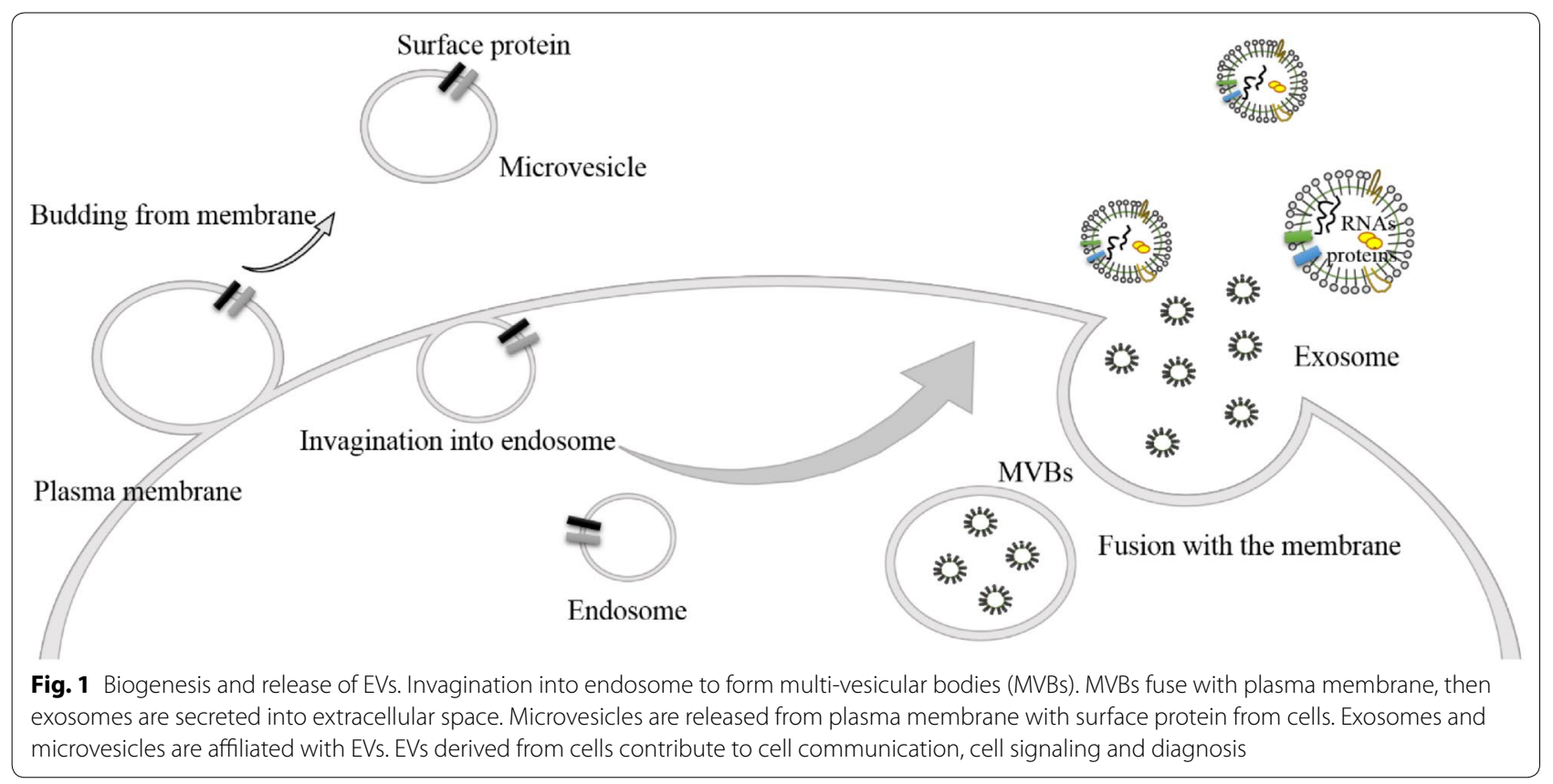

vesicles released from cardiomyocytes are called cardiosomes [17], vesicles from prostate epithelial cells are called prostatosomes [18], and vesicles released from tumor cells are called oncosomes [19].

EVs contain mRNAs and small RNAs of cells that produced them $[20,21]$. For example, microarray analysis has detected $\sim 1300$ mRNA species in EVs of the mouse mast cell line MC/9; detected mRNAs comprise $8 \%$ of total mRNA in cells of origin [21]. mRNA in MC/9 EVs is translated into polypeptides in vitro if translational machinery is supplied; i.e. the mRNA remains functional [21].

When an EV contacts a cell, their plasma membranes merge; as a result, the contents of the EV enter the cell, and can affect its function [21]. Proteins that are associated with EVs also have functions in the microenvironment. For example, the tetraspanins CD81 and CD9 immunoprecipitate together. EVs can contribute to tumor progression by facilitating angiogenesis while suppressing immune responses [22-24]. Functional proteins are released in association with EVs, so they are also involved in many neurological processes $[25,26]$.

EVs are found in most body fluids (e.g., blood, urine, saliva, amniotic fluid, semen, tears) [26-28]. An EV contains information that is related to its cell of origin, so isolating and analyzing EVs from body fluids can give important clues to diagnosis and prognosis of disease [29]. Many studies have tried to apply nucleic acids and proteins from EVs in diagnosis (Table 1). Profiles of miRNAs within serum EVs from ovarian cancer patients are significantly different than those of normal patients and of patients with benign cancer [30]. Urinary EVs may be a valuable source of diagnostically-useful miRNAs in prostate cancer [31]. Proteins in urinary EVs may provide biomarkers of acute kidney injury; for example, fetuitin-A in urinary EVs may be useful in diagnosing structural renal injury [32,33], and decrease in the level of podocalyxinlike protein 1 (PODXL) in urinary EVs may provide a biomarker to classify renal disease [34].

However, in addition to EVs, body fluids contain diverse molecular components that can impede accurate and efficient analysis [35]. For example, plasma is widely used as a specimen, but it contains abundant proteins at concentrations of $60-80 \mathrm{mg} / \mathrm{ml}[36,37]$. Furthermore, EVs are not abundant in biological fluids, so their contents are not easy to analyze [38]. Conventional isolation by ultracentrifugation recovers an average $0.21-1.08 \times 10^{8}$ particles from $200 \mu \mathrm{l}$ of plasma [39] and $1-2 \times 10^{12}$ particles from $150 \mathrm{ml}$ of cell culture medium [40]. Furthermore, the average concentration of RNA within EVs is only $10-15 \mathrm{ng}$ in $200 \mu \mathrm{l}$ plasma [28]. Altogether, the presence of other particles, the scarcity of EVs, and their contents hinder efficient analysis of EVs and complicate subsequent procedures that use them. Accordingly, to improve the yield and purity of EVs, and to ensure that subsequent analyses are not disturbed, efficient isolation of EVs is important (Fig. 2) [38, 41].

Various methods have been developed to isolate EVs effectively from biological fluids by exploiting a characteristic of EVs to separate them from coexisting particles. The methods differ in yield, purity and size distribution of isolated EVs. Therefore, high-quality EVs must be 
Table 1 Some EV applications in diagnosis

\begin{tabular}{|c|c|c|c|c|}
\hline Disease & Biospecimen & Isolation method & Biomarker & Reference \\
\hline Breast cancer & Serum & Ultracentrifugation & EpCAM & [54] \\
\hline Acute kidney injury (AKI) & Urine & Differential centrifugation & Fetuin-A & [33] \\
\hline Ovarian cancer & Serum & Density gradient centrifugation & OVCAR-3 and IGROVI & [55] \\
\hline Lung cancer & Plasma & Immunoaffinity & EGFR level & [65] \\
\hline Ovarian cancer & Serum & $\begin{array}{l}\text { Modified magnetic activated cell } \\
\text { sorting procedure }\end{array}$ & Overexpressed specific miRNAs & [30] \\
\hline Prostate cancer & Urine & Ultrafiltration & PCA3 and TMPRSS2-ETG & {$[53]$} \\
\hline $\begin{array}{l}\text { Focal segmental glomerulosclerosis } \\
\text { (FSGS) }\end{array}$ & Urine & Ultrafiltration & Podocalyxin-like protein 1 (PODXL) & {$[34]$} \\
\hline Ovarian adenoma & Serum & Size exclusion chromatography & EGFRvIII & [77] \\
\hline Prostate cancer & Urine & Polymer precipitation & Prostate specific membrane antigen (PSMA) & [85] \\
\hline Acute coronary syndromes (ACS) & Serum & Polymer precipitation & $\begin{array}{l}\text { Polygenic immunoglobulin receptors, cys- } \\
\text { tatin C(cycteine proteinase) and C5a }\end{array}$ & [81] \\
\hline Ovarian adenoma & Serum & Size exclusion chromatography & EGFRvIII & {$[77]$} \\
\hline Glioblastoma multiforme (GBM) & Serum & Microfluidics & Isocitrate dehydrogenase (IDH-1) & {$[90]$} \\
\hline Melanoma cancer & Blood & Microfluidics & MelanA & {$[33]$} \\
\hline
\end{tabular}

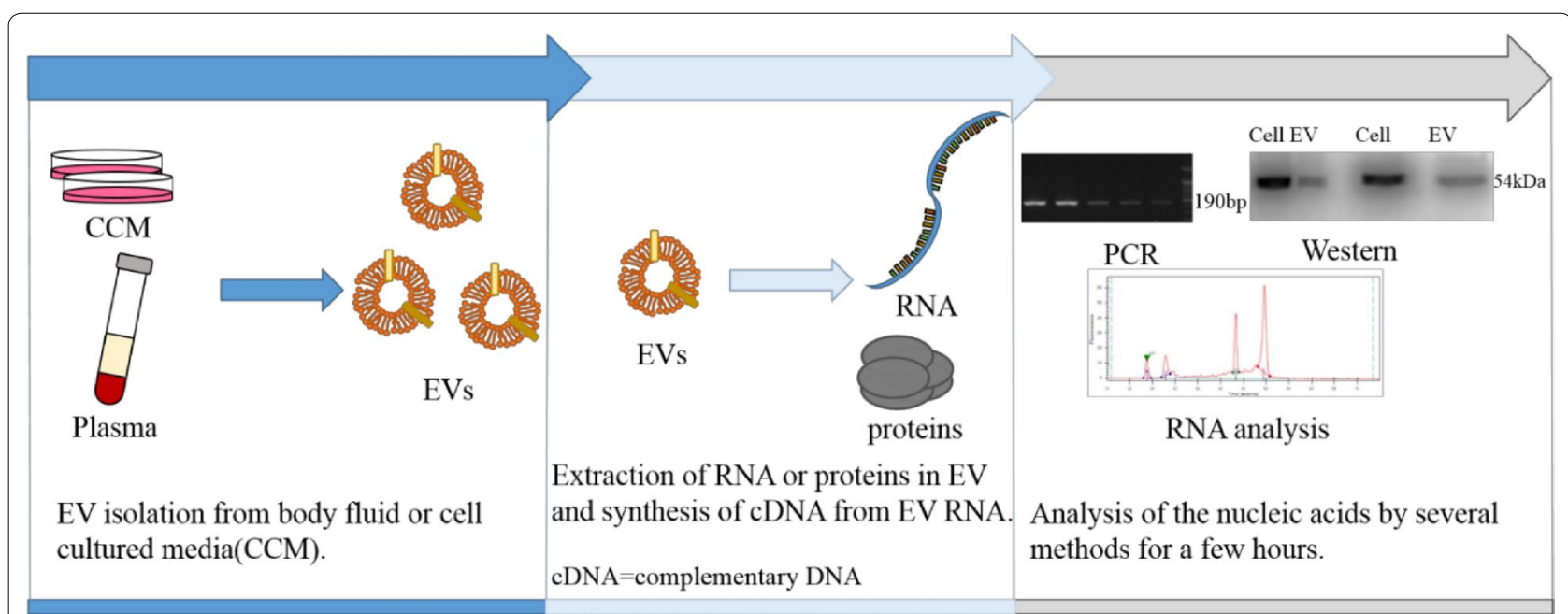

Fig. 2 Outline of the workflow for procedure for isolation and analysis of EVs. EVs are isolated from body fluid or cell culture medium (CCM). The information from EVs is obtained using PCR, western blot and RNA analysis for diagnosis

isolated using a method that is appropriate to the aim of the study, and is compatible with subsequent analyses [8, 38 ]. The following sections discuss the merits and demerits of various techniques to isolate EVs.

\section{Ultracentrifugation}

Ultracentrifugation $(\mathrm{U} / \mathrm{C})$ is a classical method that uses strong centrifugal force to isolate EVs [42, 43]. EVs sink due to the centrifugal force and form a pellet at the bottom in an ultracentrifuge tube [44]. U/C can be categorized based on principles of separation: (1) differential $\mathrm{U} / \mathrm{C}$ and (2) density gradient U/C (Fig. 3) [45-47].
Differential U/C involves serial stepwise centrifugation to remove components other than EVs: centrifugation at (1) $300 \times g$ for $10 \mathrm{~min}$ to remove live cells, (2) at $2000 \times g$ for $10 \mathrm{~min}$ to remove dead cells; (3) at $10,000 \times g$ to remove cellular debris and isolate large EVs; then finally (4) at $100,000 \times g$ to isolate exosomes (small EVs) [27, 48, 49]. This is the most-commonly-used protocol, but must be modified when the EVs are suspended in viscous fluids such as plasma or saliva [50]. The quantity of collected EVs is affected by centrifuging speed and time, so these parameters must be optimized for each rotor type. 
Cell culture medium or body fluid

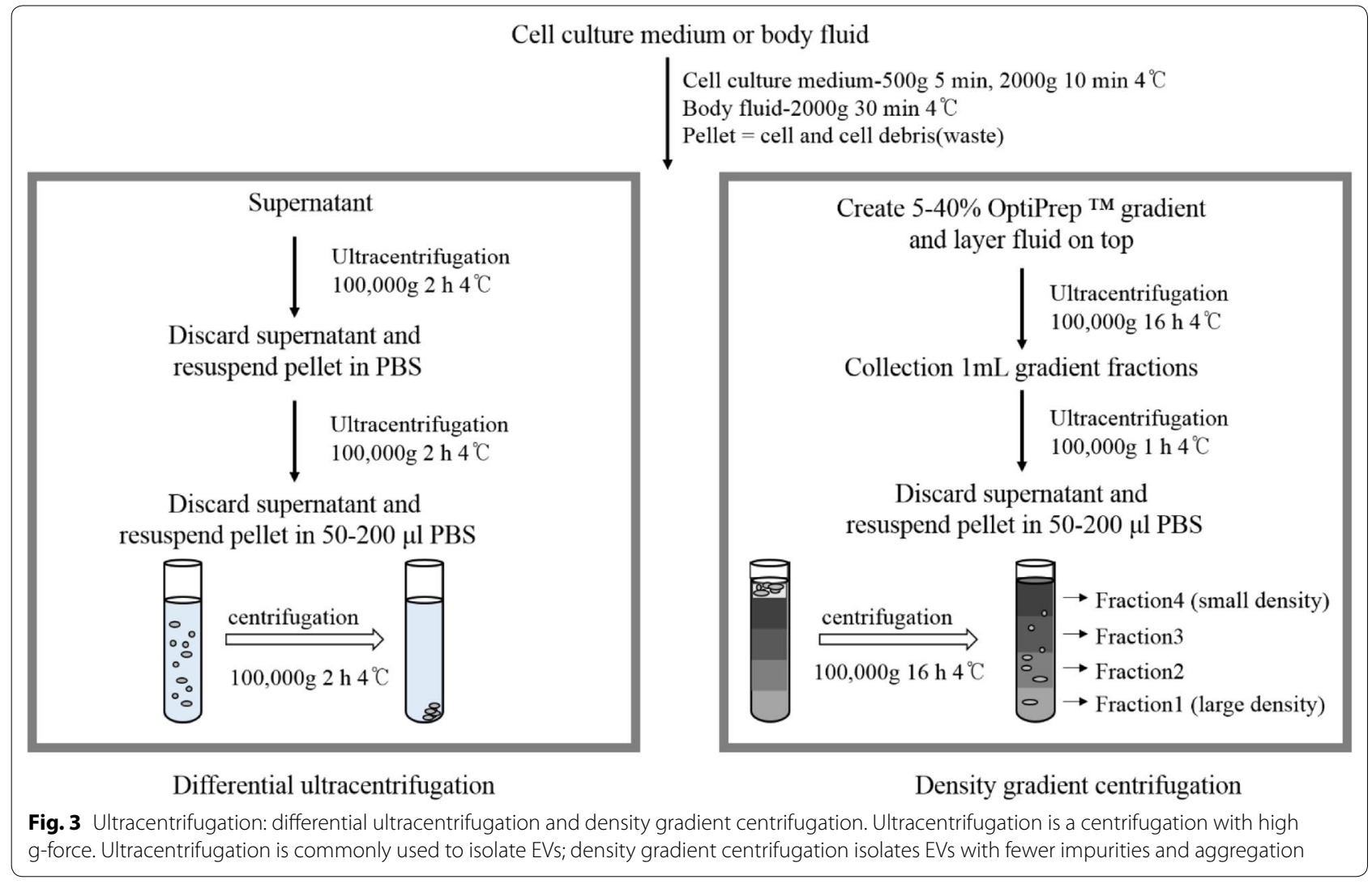

Density gradient U/C segregates EVs to specific layers according to their buoyancy in a gradient material such as sucrose, iohexol $\left(\right.$ Nycodenz $\left.^{\mathrm{TM}}\right)$ or iodixanol (Optiprep $^{\mathrm{TM}}$ ) [51]. The density fraction in which EVs accumulate is affected by gradient material $[46,52]$, and by the source of EVs [47]. For example, EVs from saliva accumulate in fractions with higher densities than EVs from conditioned medium [47].

EVs isolated by U/C have been assessed for their value as diagnostic clues. Two prostate cancer biomarkers, PCA3 and TMPRSS2-ERG, have been detected in urinary EVs from prostate cancer patients [53]. Also, urinary EVs are enriched with miRNAs, so isolation and analysis of urinary EVs can help to identify miRNA biomarkers for diseases such as prostate cancer [31]. Results can provide a diagnostic standard for comparison of patients with healthy individuals [54]. CD24 is present in serum EVs of breast-cancer patients, whereas EpCAM is not, so this difference may be a way to discriminate breast cancer from others [54]. The protein profiles in EVs of ovarian cancer cell lines OVCAR-3 and IGROV1 [55] were obtained by differential $\mathrm{U} / \mathrm{C}$ then further purification using density gradient $\mathrm{U} / \mathrm{C}(1.09-1.15 \mathrm{~g} / \mathrm{ml})$, and revealed that ovarian cancer EVs contain tissue-specific proteins that are related to tumorigenesis and metastasis.
Differential U/C has disadvantages. It is slow [50], and the presence of protein contaminants in the pellet of EVs frequently requires additional an U/C step called double pelleting; the second step reduces aggregation between EVs and proteins, but also decreases the amount of EVs isolated [56]. Another disadvantage is that protein contaminants co-occur with EVs isolated by this method, so use of a Bradford assay to estimate EV amount is not reliable $[44,51,57]$.

Differential U/C that uses high rotation speed can isolate small size vesicles, but the process can induce aggregation of vesicles and proteins [58]. Moreover, EVs isolated at high rotation speed have different phenotype, size, and even surface proteins than those isolated at low rotation speed. Due to these problems, EV analysis can have different results depending on the g-force and $\mathrm{k}$ factor of differential U/C. For example, EVs that are released from HEK293 cells are more pure when low-g force is used $(33,000 \times g$, though $67,000 \times g)$ [59].

Density gradient U/C suffers less from protein contamination (only $5-25 \%$ of $\mathrm{EV}$ concentration) than differential U/C because proteins are accumulated into different layers of density than EVs $[40,60]$. Hence, EVs isolated by density gradient centrifugation have good purity and intact morphological characteristics $[56,61]$. These 
disadvantages have been overcome by combining density gradient $\mathrm{U} / \mathrm{C}$ with other methods like filtration and chromatography. However, isolation based on size cannot discriminate EVs from other small vesicles, or among subpopulations of similarly-sized EVs.

\section{Immunoaffinity}

Immunoaffinity exploits interactions between antibodies and surface proteins of EVs to isolate EVs. Antibodies specific to surface proteins of EVs (e.g., CD9, CD81, CD63, TSG101, Alix) are linked to chemically-modified or protein-coated beads, and capture EVs by binding to these proteins $[4,62,63]$. EVs isolated based on immunoaffinity have different characteristics than those isolated based on size. Size-based separation cannot distinguish among subpopulations of EVs. For example, if the goal is to distinguish the CD81+ subpopulation of EVs from the CD63+ subpopulation, immunoaffinity is recommended [64]. Many studies have discovered EV biogenesis and subpopulations by exploiting the interaction of surface proteins with specific antibodies. Tetraspanin-specific antibodies are common in immunoaffinity [65]; for example, CD81 is internalized more slowly than CD9 [66]. ELISA with anti-CD81 antibody has been used to quantify EGFR level in plasma EVs from lung cancer patients. The EGFR exosomal proteins are possible diagnostic biomarkers in immunoaffinity methods [65].

Immunoaffinity has been incorporated into hybrid approaches that use more than one isolation method [68-69]. EVs can be isolated using magnetic beads coated with antibodies in a minimal volume of plasma [54]. Isolation of the magnetic beads enriches EVs (Fig. 4); this is a multiple technique that combines detection of EVs and proteins in one device [67]. Surface plasmon resonance imaging (SPRi) with antibody microarrays detects

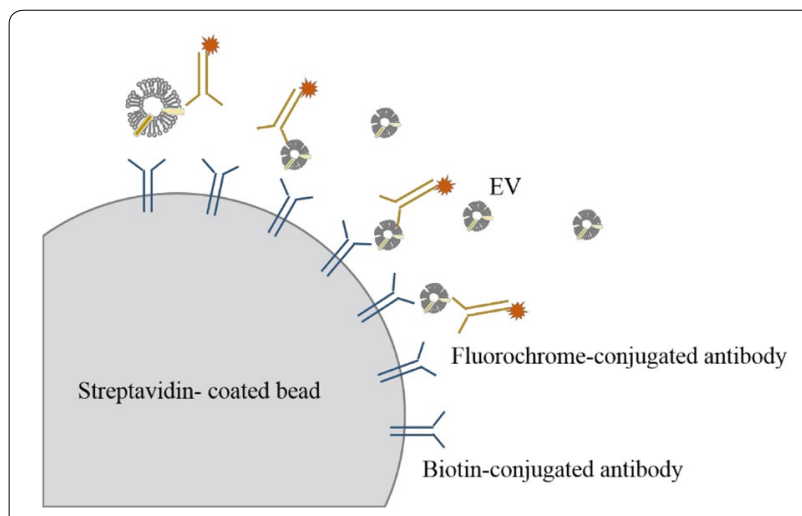

Fig. 4 Isolation method using antibody-conjugated bead. Interaction with antibody and surface protein in $\mathrm{EV}$ is a key factor in immunoaffinity. Immunoaffinity method enables identification of proteins including positive markers in EVs specific proteins on EV membranes in tumor-cell culture medium [69]. EVs isolated by the device have positive correlation with tumor cell in metastasis and prognosis prediction.

Immunoaffinity extracts more-purified EVs that bear specific identified proteins than does $\mathrm{U} / \mathrm{C}$, and achieves higher yield and greater purity of EVs than does U/C $[8$, 70]. When immunoaffinity is used to isolate EVs, subpopulations of EVs can be identified by sorting them according to their specific surface proteins. For example, $\sim 80 \%$ of A33 in EVs from cell culture medium can be captured using immunoaffinity, which achieves a yield of $\sim 25 \mu \mathrm{g}$ in a western blot assay [70]. However, antibodies are expensive, so this method is not appropriate for large samples. Moreover, for subsequent experiments, EVs must be displaced from the beads; this step may damage the EVs.

Recent studies have classified EV by size rather than by surface protein. Use of immunoaffinity does not identify the source of EV (exosome, ectosome, apoptotic body), so this method is not suitable for sorting by size. For example, exosomes, microvesicles and apoptotic bodies all have EpCAM on the membrane [8]; immunoaffinity detects the same exosomal marker in these vesicles, but they have different characteristics. Future studies should develop immunoaffinity methods to correlate surface protein identity with $\mathrm{EV}$ functions and properties.

\section{Size exclusion}

Size differences can be exploited to isolate EVs. In general, two types of size exclusion are used: filtration and chromatography (Fig. 5) [41, 71]. Filtration captures EVs on membranes, while allowing small particles like proteins to pass through them [72]. This method requires appropriate choice of pore size. Filtration is often combined with $\mathrm{U} / \mathrm{C}$ or other isolation methods $[21,56]$.

Size exclusion can concentrate EVs by using other methods, such as pressure-driven methods or U/C [40]. The pressure-driven device is suitable for samples with volumes $>400 \mathrm{ml}$ because the device is suitable for large volumes; centrifugation is appropriate for samples with volume $<400 \mathrm{ml}$ [40]. Pore size of $0.1 \mu \mathrm{m}$ is appropriate when a sonicating water bath is combined with size exclusion to isolate EVs from plasma [71]; this method revealed that EV level in plasma is lower in smokers than in non-smokers. The EV level in smokers is decreased by early apoptosis.

EVs can be isolated by combining electrophoretic migration processes and size exclusion ( $30-\mathrm{nm}$ pore size) [73]; a nanoporous membrane allows small impurities to pass through into the flow, but retains EVs larger than membrane pore size. In $30 \mathrm{~min}$, up to $65 \%$ of EVs were recovered, and up to $83.6 \%$ of protein was removed. This method is faster and achieves 14 times higher recovery 

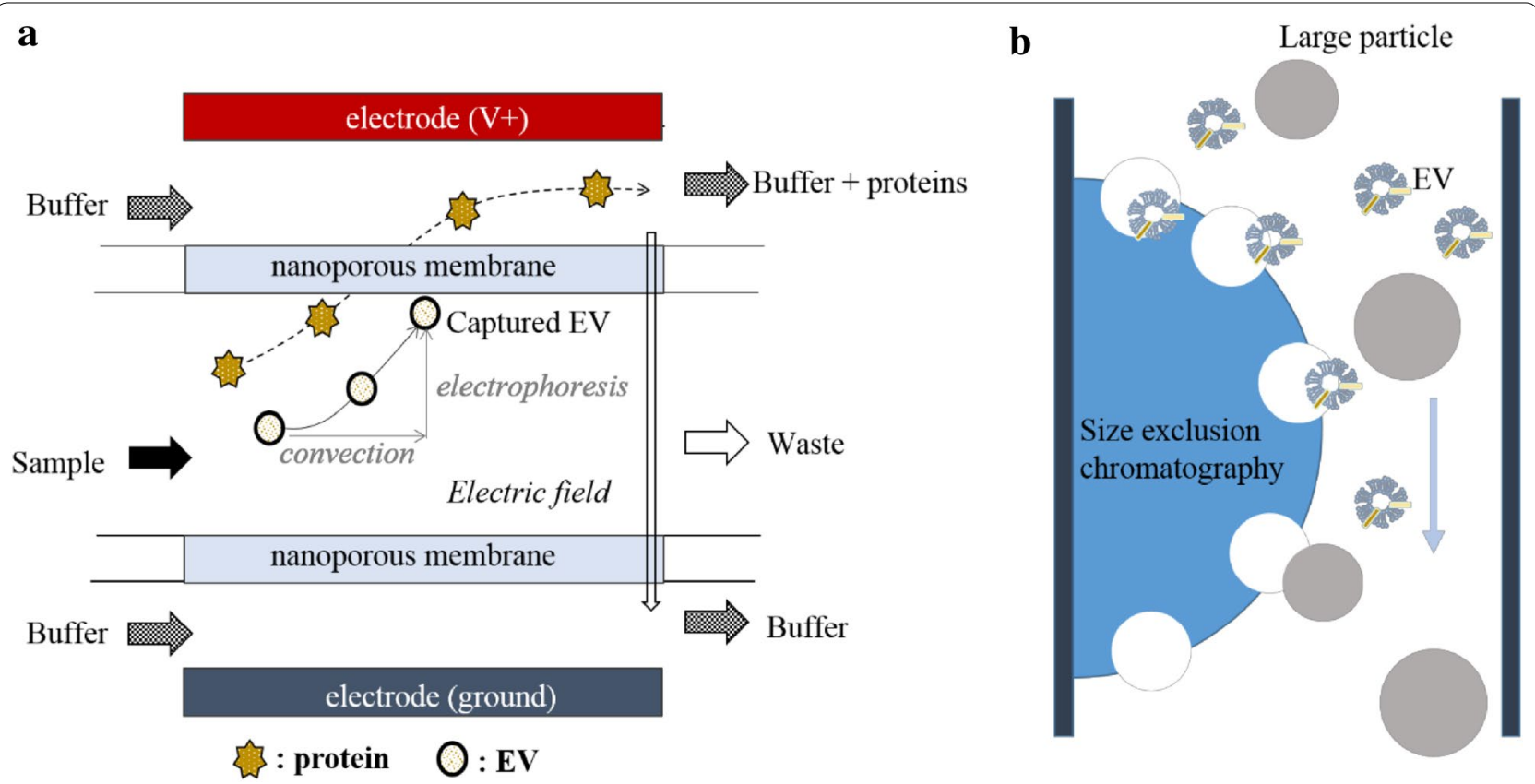

Fig. 5 Size-exclusion with (a) filter and (b) chromatography. Sorting targeted-sized EVs by size exclusion. a Filter-based isolation method considers pore size for isolation desired size vesicles. Filter-based isolation requires sorting proteins and EVs. The device with electrophoretic migration processes and size exclusion isolates EVs. b Chromatography with ultracentrifugation for isolation EVs. Small particles enter the pore and move slowly, whereas large particles pass through the column rapidly. EV-containing solution is collected

efficiency than does U/C. Size-exclusion chromatography (SEC) uses a column packed with beads that have pores smaller than EVs of concern [74]. Particles smaller than pore size enter them and move slowly, whereas particles larger than pore size pass around beads and exit the column rapidly. Fractions containing samples are eluted sequentially in order of decreasing size; the EV-containing fraction can be collected and analyzed. When SEC is used, aggregation of proteins and EVs is minimal [40, 75], and the function of EVs is usually unaffected. However, filter-based isolation and SEC separate components by their size, and therefore cannot discriminate particles that have similar size but different characteristics.

One important requirement of EV isolation is to remove high-density lipoproteins (HDL) as well as proteins. Sepharose CL-2B column in the SEC method isolates EVs of diverse size distribution in fractions [76]. EVs are highly isolated in fractions 9-12. The recovery efficiency of EVs in these fractions is ( $46 \pm 6) \%$ by NTA and (60 \pm 10$) \%$ by resistive pulse sensing (RPS). The recovery of HDL in fractions $18-20$ is ( $32 \pm 2) \%$ by APO A1. HDL in fractions $9-12$ is just $(0.65 \pm 0.3) \%$, the fractions that are isolated HDL and EVs are different.

EVs isolated by chromatography are used in diagnosis [78-79]. Ovarian adenoma can be diagnosed in serum EVs isolated by chromatographic isolation; serum EVs are analyzed using NTA and western blotting. EGFRvIII is produced by tumors, so it is detected using western blotting [77]. SEC easily isolates EVs from concentrated body fluid in one step, and has applications in diagnosis of some diseases [78].

\section{Precipitation}

\section{Polymer precipitation}

Various methods that use polymers have been developed to ease the isolation process and reduce the isolation time [80]. The methods use polymers that can precipitate or displace EVs according to surface characteristics. Exoquick $^{\mathrm{TM}}$ (System Biosciences, USA) is composed of polymers; it isolates EVs quickly and effectively by precipitation. The system just needs mixing, incubation and centrifugation; it does not require high-priced U/C or large volumes of samples [61]. Exoquick ${ }^{\mathrm{TM}}$ is efficient and is becoming adopted as an alternative to $\mathrm{U} / \mathrm{C}$, although further verification is necessary. For example, EVs are isolated by Exoquick ${ }^{\mathrm{TM}}$ from serum, then some proteins in serum EVs are analyzed using proteomics as biomarkers for acute coronary syndrome (ACS) [81]; Cystatin C and $\mathrm{C} 5 \mathrm{a}$ in $\mathrm{EV}$ isolated by $\mathrm{U} / \mathrm{C}$ and Exoquick ${ }^{\mathrm{TM}}$ are biomarkers associated with ACS in men. These proteins are demonstrated in presence of ACS.

The aqueous two phase system (ATPS) has been applied to isolate EVs (Fig. 6) [82]. After samples are completely mixed with aqueous two-phase solution 
(polyethylene glycol (PEG) and dextran), the mixture is centrifuged at $3000 \times g$ for $10-30 \mathrm{~min}$. EVs move toward the dextran phase, which has surface characteristics that are favorable to EVs [83]. ATPS is quick and easy, and does not requiring any incubation process [84]. In several biological fluids, ATPS shows higher recovery of EVs than does U/C and Exoquick ${ }^{\mathrm{TM}}$ [82]. However, as EVs are isolated in a dextran-containing solution, so the effect of dextran must be reduced before ATPS can be widely accepted. EVs isolated by ATPS have diagnostic markers. EVs purified using ATPS from prostate cancer patients are easily detected using prostate-specific membrane antigen (PSMA). ATPS isolates high-quality EVs in $\sim 15$ min. ATPS isolates uncontaminated EVs without aggregation complexes [85].

\section{Protein organic solvent precipitation}

Protein organic solvent precipitation (PROSPR) is an EV precipitation method that is a fast and simple process with organic solvents (acetone, chloroform, trichloroacetic acid) [86]. Biological fluids contain numerous proteins, soluble factors, and lipoproteins [37]. Removal of protein from biological fluids is an important requirement in EV analysis. Proteins that have hydrophilic and hydrophobic regions have dielectric strength in aqueous solution. The organic solvent is attracted to oppositelycharged amino acid residues and promote protein aggregation. The ion-pairing effect contributes to PROSPR's precipitation efficiency. Moreover, organic solvent with a salt improves protein removal [87].

PROSPR overcomes the disadvantages of U/C in which EVs are extracted with unwanted proteins, and can be damaged by high rotation speed. EVs isolated by PROSPR have only $\sim 20 \%$ as much protein contamination than those isolated by U/C. Compared to ultracentrifugationcushion, PROSPR can isolate EVs with EV-associated protein by 7.2 times [88].

\section{Microfluidics techniques}

EVs can be isolated by microfluidic devices that induce flow of liquids within micro-sized channels [38, 89]. Microfluidic devices are small but purify and separate faster than other isolation methods. Microfluidic devices require only a small sample, so the cost, processing time, and consumption of reagents are reduced. EVs isolated using microfluidic devices tend to retain their morphology. Microfluidics techniques capture EVs by using immunoaffinity, by sieving, or by trapping them in porous structures [90].

Microfluidic devices have been combined with immunoaffinity methods to detect diagnostic markers. EVs are captured by binding to specific antibodies on channels or by passing through membranes (Fig. 7a). Modified microfluidics can isolate EVs from 100-400 $\mu \mathrm{l}$ serum samples and brain tumor specimens [90]; modified surfaces are biotinylated anti-63, control IgG and anti-CD4. The antibodies bind specific EVs because EVs secreted by tumor cells have surface specific markers. Antibodies coated on the surface of device increase the efficiency of EV capture.

EVs protect RNA from RNase in body fluids, which otherwise degrade it rapidly [91]; therefore RNAs in EV are stable during RNA analysis, so RNA in EVs can be analyzed to detect biomarkers for diagnosis. RNA level

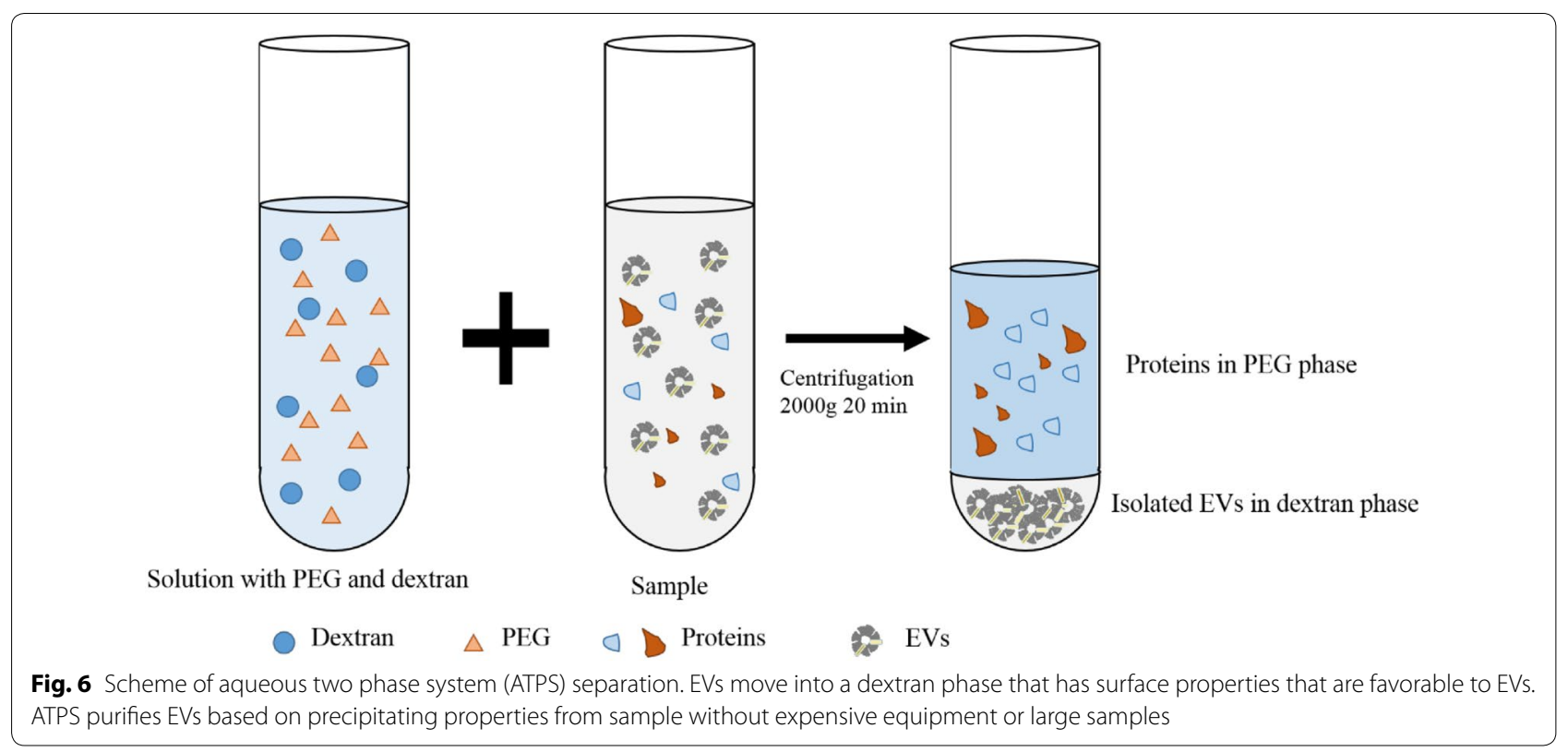




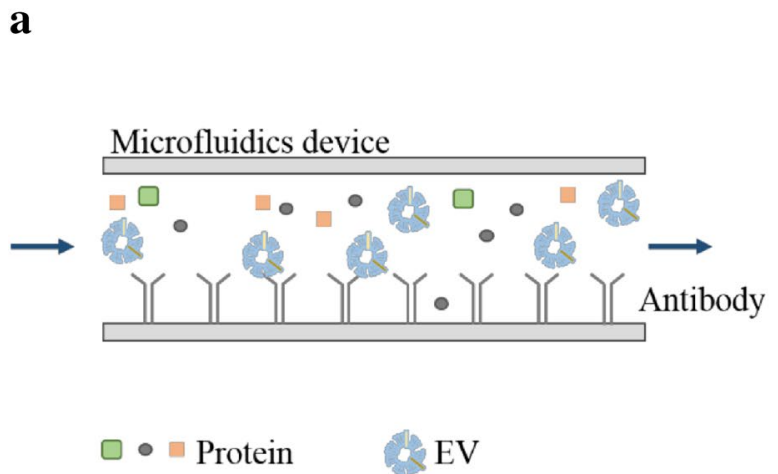

$\square \cdot \square$ Protein $\quad B \mathrm{EV}$ b

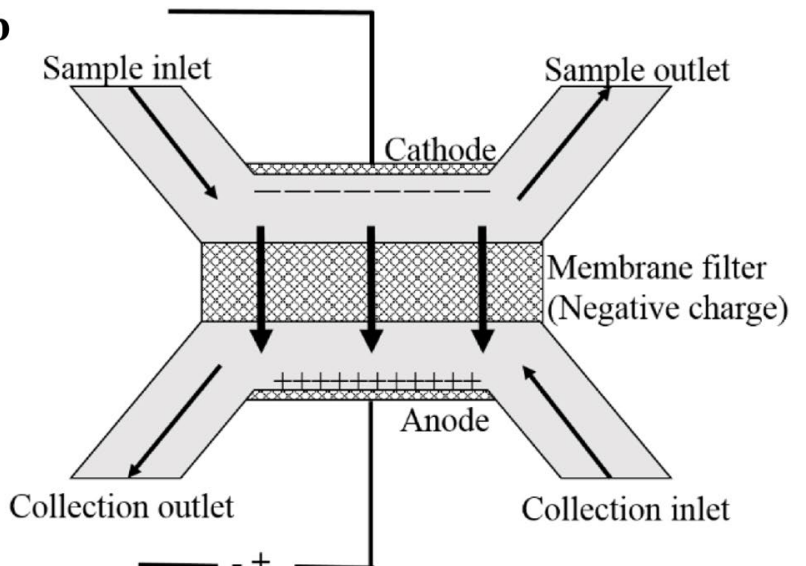

St

$-+$

Fig. 7 Microfluidic system to isolate EVs. Microfluidics techniques developed for EV purification are classified into immunoaffinity approach, sieving EVs, and trapping EVs with porous structures. a Capture of EVs occurs by binding to specific antibodies on channels. The device with antibody increases EV-capture efficiency. b Microfluidics with filter pore size $~ 500 \mathrm{~nm}$ isolate EVs without aggregation. EVs have negatively charged phospholipid membranes and therefore move across filter membranes

in EVs differs between glioblastoma multiforme (GBM) patients and healthy individuals; real-time polymerase chain reaction (RT-PCR) and RNA sequencing of mRNA in EVs from GBM patients detected mRNA of mutated IDH-1. The recovery of EVs was $42-94 \%$ at the RNA level [90]. Microfluidic devices can sort specific EVs, and enable can diagnoses disease from a small sample in a single step [92].

Microfluidic devices with filter membranes can purify EVs like small vesicles (Fig. 7b) [93]. A microfluidics device that includes a filter membrane that uses porous polymer monoliths (PPM) can isolate EVs from mouse blood; the membrane removes cells and cell debris and allows passage of small vesicles like EVs for isolation and diagnosis.

Filtration can be classified as pressure-driven or electrophoresis-driven [93]. Pressure-driven filtration uses a syringe pump; filter membranes with $500-\mathrm{nm}$ pores are usually used because EVs can clog the filter if pores are smaller than this. Electrophoresis-driven filtration exploits the negatively-charged phospholipid membranes of EVs to move them across filter membranes, whereas proteins and other molecules have different charges due to side chains of amino acids. EVs cross the filter membrane and are isolated into a collection solution. Electrophoresis-driven filtration is not blocked by any molecule, but the flow is sometimes hindered by gas bubbles that form at output $>0.7 \mathrm{~V} / \mathrm{cm}$.

A microfluidics device with ciliated micropillars purifies EVs without contamination [94]. Ciliated micropillars on the wall filter lipid vesicles like EVs on several scales. Intact EVs are trapped in nanowires, whereas large vesicles like cells and cell debris, and small molecules like proteins are passed in a continuous flow. Soaking micropillars in PBS for $24 \mathrm{~h}$ releases the trapped EVs by dissolving the vesicles. A quantum dot (QD) directly visualizes the trapped EVs on micropillars; the QD is not dissolved in PBS and not carried with EVs.

EV purification using microfluidic systems is still in its early stages of development, but many microfluidic devices have demonstrated that it is an effective method to isolate EVs. Microfluidic devices can damage EVs due to shear stress, and require macro-scale samples [92]. Microfluidics engineers should collaborate with biologists and clinicians to develop techniques that are suitable for EV recovery. Microfluidic devices to isolate EVs could be used in clinics.

\section{Conclusion}

Although EVs have potential applications in diagnosis and therapy of diseases, points for improvement should be noted. According to purpose, the choice of a suitable isolation method increases the effectiveness of isolation. For example, immunoaffinity can detect specific EVs but is not appropriate for large-volume samples [50]. In acute clinical usage, the method must be fast and inexpensive for use in emergencies. Also, a purification process must isolate intact EVs. The genetic information in EVs is generally unstable [95], so damaged EVs may not be useful in precise experiments.

Many studies have used EVs in disease diagnosis, disease monitoring and predictive tracking [96, 97]. The advantage of diagnosis using EVs is that they can be isolated from body fluids by minimally invasive biopsy [65]. Minimalized invasive diagnosis is preferable to traditional invasive diagnosis which causes bleeding and pain 
[98]. Moreover, EVs also can be used as vehicles for drug delivery in disease therapy [99]. Stem cell-derived EVs can induce tissue regeneration as a cell-free approach. Taken together, clinical application of EV analysis will be expanded, but much research remains to be completed. EVs focus on individual biologics to strengthen the knowledge in the field. Continued search will identify EV-based diagnostic and prognostic biomarkers, and will improve understanding of the mechanisms of disease pathogenesis.

\section{Authors' contributions}

HK and JK summarized previous works and wrote the manuscript. JP provided advice for the manuscript. All authors read and approved the final manuscript.

\section{Author details \\ ${ }^{1}$ School of Interdisciplinary Bioscience and Bioengineering, POSTECH, 77 Cheongam-Ro, Pohang, Gyeongbuk 37673, Republic of Korea. ${ }^{2}$ Department of Mechanical Engineering, POSTECH, 77 Cheongam-Ro, Pohang, Gyeongbuk 37673 , Republic of Korea}

\section{Competing interests}

We confirm that I have read SpringerOpen's guidance on competing interests and have included a statement indicating that none of the authors have any competing interests in the manuscript.

\section{Funding}

This research was supported by a grant of the Korea Health Technology R\&D Project through the Korea Health Industry Development Institute (KHIDI), funded by the Ministry of Health and Welfare, Republic of Korea (Grant no. HI16C0665).

Received: 14 November 2016 Accepted: 13 February 2017

Published online: 01 March 2017

\section{References}

1. Trams EG et al (1981) Exfoliation of membrane ecto-enzymes in the form of micro-vesicles. Biochimica et Biophysica Acta (BBA)-Biomembr 645(1):63-70

2. Ingato D et al (2016) Good things come in small packages: Overcoming challenges to harness extracellular vesicles for therapeutic delivery. J Control Release. 241:174-185

3. Momen-Heravi F et al (2013) Current methods for the isolation of extracellular vesicles. Biol Chem 394(10):1253-1262

4. Akers JC et al (2013) Biogenesis of extracellular vesicles (EV): exosomes, microvesicles, retrovirus-like vesicles, and apoptotic bodies. J Neurooncol 113(1):1-11

5. Raposo G, Stoorvogel W (2013) Extracellular vesicles: exosomes, microvesicles, and friends. J Cell Biol 200(4):373-383

6. van Niel G et al (2006) Exosomes: a common pathway for a specialized function. J Biochem 140(1):13-21

7. Redman C et al (2012) Review: does size matter? Placental debris and the pathophysiology of pre-eclampsia. Placenta 33:S48-\$54

8. Tauro BJ et al (2012) Comparison of ultracentrifugation, density gradient separation, and immunoaffinity capture methods for isolating human colon cancer cell line LIM1863-derived exosomes. Methods 56(2):293-304

9. Heijnen HF et al (1999) Activated platelets release two types of membrane vesicles: microvesicles by surface shedding and exosomes derived from exocytosis of multivesicular bodies and-granules. Blood 94(11):3791-3799

10. Mørk M et al (2016) Preanalytical, analytical, and biological variation of blood plasma submicron particle levels measured with nanoparticle tracking analysis and tunable resistive pulse sensing. Scand J Clin Lab Investig. 76:1-12
11. Budnik V, Ruiz-Cañada C, Wendler F (2016) Extracellular vesicles round off communication in the nervous system. Nat Rev Neurosci 17(3):160-172

12. Andreu Z, Yáñez-Mó M (2014) Tetraspanins in extracellular vesicle formation and function. Front Immunol 5:442

13. Muralidharan-Chari V et al (2009) ARF6-regulated shedding of tumor cellderived plasma membrane microvesicles. Curr Biol 19(22):1875-1885

14. Andaloussi SE et al (2013) Extracellular vesicles: biology and emerging therapeutic opportunities. Nat Rev Drug Discovery 12(5):347-357

15. Gould SJ, Raposo G (2013) As we wait: coping with an imperfect nomenclature for extracellular vesicles. J Extracell Vesicles 2. doi:10.3402/jev. v2i0.20389

16. Cocucci E, Racchetti G, Meldolesi J (2009) Shedding microvesicles: artefacts no more. Trends Cell Biol 19(2):43-51

17. Waldenström A et al (2012) Cardiomyocyte microvesicles contain DNA/RNA and convey biological messages to target cells. PLOS ONE 7(4):e34653

18. Gusachenko O, Zenkova M, Vlassov V (2013) Nucleic acids in exosomes: disease markers and intercellular communication molecules. Biochemistry (Moscow) 78(1):1-7

19. Minciacchi VR, Freeman MR, Di Vizio D (2015) Extracellular vesicles in cancer: exosomes, microvesicles and the emerging role of large oncosomes. In Seminars in cell developmental biology. Elsevier, Amsterdam

20. Lee Y, Andaloussi SE, Wood MJ (2012) Exosomes and microvesicles: extracellular vesicles for genetic information transfer and gene therapy. Hum Mol Genet 21(R1):R125-R134

21. Valadi $\mathrm{H}$ et al (2007) Exosome-mediated transfer of mRNAs and microRNAs is a novel mechanism of genetic exchange between cells. Nat Cell Biol 9(6):654-659

22. Simons M, Raposo G (2009) Exosomes-vesicular carriers for intercellular communication. Curr Opin Cell Biol 21(4):575-581

23. Denzer $\mathrm{K}$ et al (2000) Exosome: from internal vesicle of the multivesicular body to intercellular signaling device. J Cell Sci 113(19):3365-3374

24. Park JE et al (2010) Hypoxic tumor cell modulates its microenvironment to enhance angiogenic and metastatic potential by secretion of proteins and exosomes. Mol Cell Proteom 9(6):1085-1099

25. Lässer C et al (2011) Human saliva, plasma and breast milk exosomes contain RNA: uptake by macrophages. J Transl Med 9(1):1

26. Admyre C et al (2007) Exosomes with immune modulatory features are present in human breast milk. J Immunol 179(3):1969-1978

27. Revenfeld ALS et al (2014) Diagnostic and prognostic potential of extracellular vesicles in peripheral blood. Clin Ther 36(6):830-846

28. Gallo A et al (2012) The majority of microRNAs detectable in serum and saliva is concentrated in exosomes. PLoS ONE 7(3):e30679

29. Pant S, Hilton H, Burczynski ME (2012) The multifaceted exosome: biogenesis, role in normal and aberrant cellular function, and frontiers for pharmacological and biomarker opportunities. Biochem Pharmacol 83(11):1484-1494

30. Taylor DD, Gercel-Taylor C (2008) MicroRNA signatures of tumor-derived exosomes as diagnostic biomarkers of ovarian cancer. Gynecol Oncol 110(1):13-21

31. Bryzgunova OE et al (2016) Comparative study of extracellular vesicles from the urine of healthy individuals and prostate cancer patients. PLoS ONE 11(6):e0157566

32. du Cheyron $\mathrm{D}$ et al (2003) Urinary measurement of $\mathrm{Na}+/ \mathrm{H}+$ exchanger isoform 3 (NHE3) protein as new marker of tubule injury in critically ill patients with ARF. Am J Kidney Dis 42(3):497-506

33. Zhou H et al (2006) Exosomal Fetuin-A identified by proteomics: a novel urinary biomarker for detecting acute kidney injury. Kidney Int 70(10):1847-1857

34. Cheruvanky A et al (2007) Rapid isolation of urinary exosomal biomarkers using a nanomembrane ultrafiltration concentrator. Am J PhysiologyRenal Physiology 292(5):F1657-F1661

35. Welton JL et al (2015) Ready-made chromatography columns for extracellular vesicle isolation from plasma. J Extracell Vesicles 4. doi:10.3402/ jev.v4.27269

36. Rekker K et al (2014) Comparison of serum exosome isolation methods for microRNA profiling. Clin Biochem 47(1):135-138

37. Kalra $\mathrm{H}$ et al (2013) Comparative proteomics evaluation of plasma exosome isolation techniques and assessment of the stability of exosomes in normal human blood plasma. Proteomics 13(22):3354-3364 
38. Kanwar SS et al (2014) Microfluidic device (ExoChip) for on-chip isolation, quantification and characterization of circulating exosomes. Lab Chip 14(11):1891-1900

39. Huang $X$ et al (2013) Characterization of human plasma-derived exosomal RNAs by deep sequencing. BMC Genom 14(1):1

40. Lobb RJ et al (2015) Optimized exosome isolation protocol for cell culture supernatant and human plasma. J Extracell Vesicles 4:27031. doi:10.3402/ jev.v4.27031

41. Merchant ML et al (2010) Microfiltration isolation of human urinary exosomes for characterization by MS. PROTEOMICS-Clin Appl 4(1):84-96

42. van der Pol E et al (2012) Classification, functions, and clinical relevance of extracellular vesicles. Pharmacol Rev 64(3):676-705

43. Mathivanan S et al (2012) ExoCarta 2012: database of exosomal proteins, RNA and lipids. Nucleic Acids Res 40(D1):D1241-D1244

44. Ismail $\mathrm{N}$ et al (2013) Macrophage microvesicles induce macrophage differentiation and miR-223 transfer. Blood 121(6):984-995

45. Graham JM (2001) Isolation of Golgi membranes from tissues and cells by differential and density gradient centrifugation. Current Protoc Cell Biol 121:3.9.1-3.9.24

46. Brasaemle DL, Wolins NE (2006) Isolation of lipid droplets from cells by density gradient centrifugation. Curr Protoc Cell Biol 3.15.1-3.15.12. doi:10.1002/0471143030.cb0315s29

47. Iwai K et al (2016) Isolation of human salivary extracellular vesicles by iodixanol density gradient ultracentrifugation and their characterizations. J Extracell Vesicles. doi:10.3402/jev.v5.30829

48. Eitan E et al (2015) Extracellular vesicle-depleted fetal bovine and human sera have reduced capacity to support cell growth. J Extracell Vesicles 4:26373. doi:10.3402/jev.v4.26373

49. Friedhoff AJ, Winkle EV (1962) Isolation and characterization of a compound from the urine of schizophrenics. Nature 194:897-898

50. Théry C et al (2006) Isolation and characterization of exosomes from cell culture supernatants and biological fluids. Curr Protoc Cell Biol 194:3.22.1-3.22.29

51. Cvjetkovic A, Lötvall J, Lässer C (2014) The influence of rotor type and centrifugation time on the yield and purity of extracellular vesicles. J Extracell Vesicles 3. doi:10.3402/jev.v3.23111

52. Sims NR, Anderson MF (2008) Isolation of mitochondria from rat brain using Percoll density gradient centrifugation. Nat Protoc 3(7):1228-1239

53. Nilsson J et al (2009) Prostate cancer-derived urine exosomes: a novel approach to biomarkers for prostate cancer. Br J Cancer 100(10):1603-1607

54. Rupp A-K et al (2011) Loss of EpCAM expression in breast cancer derived serum exosomes: role of proteolytic cleavage. Gynecol Oncol 122(2):437-446

55. Liang B et al (2013) Characterization and proteomic analysis of ovarian cancer-derived exosomes. Journal of proteomics 80:171-182

56. Witwer KW et al (2013) Standardization of sample collection, isolation and analysis methods in extracellular vesicle research. J Extracell Vesicles 2. doi:10.3402/jev.v2i0.20360

57. Barenholz Y et al (1977) A simple method for the preparation of homogeneous phospholipid vesicles. Biochemistry 16(12):2806-2810

58. Linares R et al (2015) High-speed centrifugation induces aggregation of extracellular vesicles. J Extracell Vesicles 4. doi:10.3402/jev.v4.29509

59. Jeppesen DK et al (2014) Comparative analysis of discrete exosome fractions obtained by differential centrifugation. J Extracell Vesicles 3:25011. doi:10.3402/jev.v3.25011

60. Webber J, Clayton A (2013) How pure are your vesicles? J Extracell Vesicles 2(1). doi:10.3402/jev.v2i0.19861

61. Yamada T et al (2012) Comparison of methods for isolating exosomes from bovine milk. J Vet Med Sci 74(11):1523-1525

62. Kosaka $\mathrm{N}$ et al (2010) Secretory mechanisms and intercellular transfer of microRNAs in living cells. J Biol Chem 285(23):17442-17452

63. Lässer C, Eldh M, Lötvall J (2012) Isolation and characterization of RNAcontaining exosomes. JoVE 59:e3037-e3037

64. Oksvold MP et al (2014) Expression of B-cell surface antigens in subpopulations of exosomes released from B-cell lymphoma cells. Clin Ther 36(6):847-862

65. Yamashita T et al (2013) Epidermal growth factor receptor localized to exosome membranes as a possible biomarker for lung cancer diagnosis. Die Pharmazie-Int J Pharm Sci 68(12):969-973
66. Abache T et al (2007) The transferrin receptor and the tetraspanin web molecules CD9, CD81, and CD9P-1 are differentially sorted into exosomes after TPA treatment of K562 cells. J Cell Biochem 102(3):650-664

67. He M et al (2014) Integrated immunoisolation and protein analysis of circulating exosomes using microfluidic technology. Lab Chip 14(19):3773-3780

68. Wei F, Yang J, Wong DT (2013) Detection of exosomal biomarker by electric field-induced release and measurement (EFIRM). Biosens Bioelectron 44:115-121

69. Zhu L et al (2014) Label-free quantitative detection of tumor-derived exosomes through surface plasmon resonance imaging. Anal Chem 86(17):8857-8864

70. Mathivanan S et al (2010) Proteomics analysis of A33 immunoaffinitypurified exosomes released from the human colon tumor cell line LIM1215 reveals a tissue-specific protein signature. Mol Cell Proteom 9(2):197-208

71. Grant R et al (2011) A filtration-based protocol to isolate human plasma membrane-derived vesicles and exosomes from blood plasma. J Immunol Methods 371(1):143-151

72. Ohshima K et al (2010) Let-7 microRNA family is selectively secreted into the extracellular environment via exosomes in a metastatic gastric cancer cell line. PLoS ONE 5(10):e13247

73. Cho $S$ et al (2016) Isolation of extracellular vesicle from blood plasma using electrophoretic migration through porous membrane. Sens Actuators B: Chem 233:289-297

74. Taylor DD, Shah S (2015) Methods of isolating extracellular vesicles impact down-stream analyses of their cargoes. Methods 87:3-10

75. Nordin JZ et al (2015) Ultrafiltration with size-exclusion liquid chromatography for high yield isolation of extracellular vesicles preserving intact biophysical and functional properties. Nanomed Nanotechnol Biol Med 11(4):879-883

76. Böing AN, et al (2014) Single-step isolation of extracellular vesicles by size-exclusion chromatography. J Extracell Vesicles 3. doi:10.3402/jev. v3.23430

77. Gercel-Taylor C et al (2012) Nanoparticle analysis of circulating cellderived vesicles in ovarian cancer patients. Anal Biochem 428(1):44-53

78. Lozano-Ramos I et al (2015) Size-exclusion chromatography-based enrichment of extracellular vesicles from urine samples. J Extracell Vesicles 4:27369. doi:10.3402/jev.v4.27369

79. Rabinowits $\mathrm{G}$ et al (2009) Exosomal microRNA: a diagnostic marker for lung cancer. Clin Lung Cancer 10(1):42-46

80. Lewis GD, Metcalf TG (1988) Polyethylene glycol precipitation for recovery of pathogenic viruses, including hepatitis A virus and human rotavirus, from oyster, water, and sediment samples. Appl Environ Microbiol 54(8):1983-1988

81. de Hoog VC et al (2013) Serum extracellular vesicle protein levels are associated with acute coronary syndrome. Eur Heart J: Acute Cardiovasc Care 2(1):53-60

82. Shin $\mathrm{H}$ et al (2015) High-yield isolation of extracellular vesicles using aqueous two-phase system. Scientific Rep 5. doi:10.1038/srep13103

83. Walter H (2012) Partitioning in aqueous two-phase system: theory, methods, uses, and applications to biotechnology. Elsevier, Amsterdam

84. Kim J et al (2015) Isolation of high-purity extracellular vesicles by extracting proteins using aqueous two-phase system. PLOS ONE 10(6):e0129760

85. Park YH, Shin HW, Jung AR, Kwon OS, Choi YJ, Park J, Lee JY (2016) Prostate-specific extracellular vesicles as a novel biomarker in human prostate cancer. Scientific Rep 6. doi:10.1038/srep30386

86. Gallart-Palau X, et al (2015) Extracellular vesicles are rapidly purified from human plasma by PRotein Organic Solvent PRecipitation (PROSPR). Scientific Rep 5. doi:10.1038/srep14664

87. Crowell AM, Wall MJ, Doucette AA (2013) Maximizing recovery of water-soluble proteins through acetone precipitation. Anal Chim Acta 796:48-54

88. Gallart-Palau X, Serra A, Sze SK (2016) Enrichment of extracellular vesicles from tissues of the central nervous system by PROSPR. Mol Neurodegener 11(1):1

89. Whitesides GM (2006) The origins and the future of microfluidics. Nature 442(7101):368-373

90. Chen C et al (2010) Microfluidic isolation and transcriptome analysis of serum microvesicles. Lab Chip 10(4):505-511 
91. Houseley J, LaCava J, Tollervey D (2006) RNA-quality control by the exosome. Nat Rev Mol Cell Biol 7(7):529-539

92. Liga A et al (2015) Exosome isolation: a microfluidic road-map. Lab Chip 15(11):2388-2394

93. Davies RT et al (2012) Microfluidic filtration system to isolate extracellular vesicles from blood. Lab Chip 12(24):5202-5210

94. Wang Z et al (2013) Ciliated micropillars for the microfluidic-based isolation of nanoscale lipid vesicles. Lab Chip 13(15):2879-2882

95. Haile S, Estévez AM, Clayton C (2003) A role for the exosome in the in vivo degradation of unstable mRNAs. Rna 9(12):1491-1501
96. Silva J et al (2012) Analysis of exosome release and its prognostic value in human colorectal cancer. Genes Chromosom Cancer 51(4):409-418

97. Cortez MA, Calin GA (2009) MicroRNA identification in plasma and serum: a new tool to diagnose and monitor diseases. Expert Opin Biol Ther 9(6):703-711

98. Verma M et al (2015) Extracellular vesicles: potential applications in cancer diagnosis, prognosis, and epidemiology. BMC Clin Pathol 15(1):6

99. van Dommelen SM et al (2012) Microvesicles and exosomes: opportunities for cell-derived membrane vesicles in drug delivery. J Control Release 161(2):635-644

\section{Submit your manuscript to a SpringerOpen ${ }^{\circ}$ journal and benefit from:}

- Convenient online submission

- Rigorous peer review

- Immediate publication on acceptance

- Open access: articles freely available online

- High visibility within the field

- Retaining the copyright to your article 